\title{
A Method of Extended Testability Modeling Based on Fault Injection System
}

\author{
Li Zhiyu ${ }^{1, \text { a }}$, Huang Kaoli², Lian Guangyao ${ }^{2}$ \\ ${ }^{1}$ Ordnance Engineering College, Shi Jiazhuang, China \\ 2,Ordnance Technology School, Shi Jiazhuang, China \\ abryantzhi@126.com
}

Keywords: Fault injection; testability modeling; on-off multi-state system; extended D matrix; extended diagnostic tree

\begin{abstract}
Presently, the slowly development of DFT is mainly due to the lack of effective testability validation. The validation is an important part of the DFT; it will accurately detect equipment Fault Detection Rate (FDR), Fault Isolation Rate (FIR), Fault Alarm Rate (FAR) and make sure other important indicators of the test. Fault injection techniques have become the core of the DFT validation. It directly introduces faults (hardware or software) into the target equipment systems by means of artificial. Thereby it reduces the failure of the incubation period, and accelerates the process of system failure. Testability-modeling is an effective method if fault injection. The existing methods for testability-modeling have some constraints, including the consistency of failure criterion, failure transmitting and logical-value criterion of test. Aiming at states differences of on-off multi-state system, the method of extended testability modeling was proposed. Failure, test, D matrix and diagnostic tree were defined extended with the introduction of states, and an extended testability model was defined also. The algorithms for extended testability modeling and analysis were described, which include modeling, generations of extended D matrix and extended diagnostic tree. A hydraulic control system is taken as an example for the engineering application, which shows that this method is feasible and effective.
\end{abstract}

\section{Introduction}

On-off multi-state system is a special system which is configuration with a number of on-off children systems, and the state has significant differences. When using the traditional analysis to this system has the following difficult problems: (1) In different state, the meanings of elements' faults of system are different; (2) In different state, the transfer relationships of elements within the system are different; (3) In different state, the system logic value criterion of the system are different.

To solve these problems, this paper gives an expanding Testability-modeling method which can be used in on-off multi-state system. Based on introduction of the set of states, it expands definition of the fault, test, D matrix and the diagnostic tree, and gives expanding Testability-modeling algorithm. And finally, it checks its good availability and affection by typical case of application of on-off multi-state system.

\section{Problems Analysis}

Xen3.0 structure diagram is shown as an appendix (Figure. 2) at the end of the paper.

\section{Existing Testability-modeling constraints}

In existing Testability-modeling test methods, there are constraints as follows:

1) Consistency of failure criterion

In Testability-modeling, determining the underlying unit within the system failure is needed. The meaning of these units failed must be constant in the process of the work in the system, that is, it's consistency of failure criterion.

2) Consistency of failure

For the test model, along the direction of signal flow, fault can pass through the bottom cell, that 
is the bottom unit of work status or fault conditions will not affect the failure transmission. The failure transfer relationship has consistency.

3) Consistency of logical value criterion

111's results are treated as two-valued logic: Logic 0 indicates the test result is normal; Logic 1 indicates that the test results fail. The meaning of the logical values of these tests in the course of the work must be constant, that is, the value of the test criteria has consistency.

\section{Characteristics and problems}

In the on-off multi-state system, usually there are one or more on-off control units. That these units are ON or OFF can control the system working state. It has the following characteristics:

1) The on-off control unit itself will change the failure criterion

In the system working process, for switching the working status, the operating mode of on-off controlling unit should be changed. For example, switch from connecting mode to blocking mode, or in the reverse. In connecting mode, its main failure is blocked; In blocking mode, its main failure is connecting. Therefore, the function failure criteria of the on-off control unit in different operating mode are different. Its failure criteria in the course of system work will change with the system operating mode conversion.

2) Failure transition relationship will change

When the on-off control unit is in connecting mode, its input signal can be passed to the unit's output. The front signal flow can transmit to backward through the on-off control unit. On reverse, when the on-off control unit is in blocking mode, its input signal cannot be passed to the unit's output. And the front signal flow cannot transmit to backward. Therefore, during the system working process, the failures transmitting relationship will change in different working state.

3) Logical value criteria of testing will change

In system designing, usually test signal which is easy to be obtained. Compare signal test value with the threshold to determine the results normal or fault. When there is an on-off control unit in the signal path, its working mode transition will lead to the apparent magnitude change of the signal after it, and using single test threshold will lead to miscarriage of justice. Therefore, in the system work process should set a number of logical value criterion based on different testing need.

\section{Definition of the extended test model}

Definition 1. Set of states. State set is defined as

$S=\left\{s_{k} \mid k=1,2, \ldots, K\right\}$

Where $S$ is the set of states; $s_{k}$ is the $k$-th system state; $K$ is the total number of states.

Definition 2. Extension failure. The extension failure is defined as the followed:

$E F=(F, F C)$

Where $E F$ is the extension failure; $F$ is the fault set, and $F=\left\{f_{i} \mid i=1,2, \ldots, I\right\}, f_{i}$ is the $i$-th level of the agreed unit failure, $I$ is the total number of units; $F C$ is failure criterion collection, and $F C=\{f c$ $\left.{ }_{i k} \mid i=1,2, \ldots, I ; k=1,2, \ldots, K\right\}, f c_{i k}$ is the $i$-th unit failure criterion in the $k$-th failure mode.

Definition 3. Extension test. Extension test of the dual group is defined as follows:

$E T=(T, T C)$

Where $E T$ is the extension test; $T$ is the testing set, and $T=\left\{t_{j} \mid j=1,2, \ldots, J\right\}, t_{j}$ is the $j$-th test, $J$ is the total number of tests; $T C$ is logic criterion collection for the test, and $T C=\left\{t c_{j k} \mid j=1,2, \ldots, J ; k=1,2, \ldots, K\right\}, t c_{j k}$ is the $j$-th test logical value criterion in the $k$-th state.

Definition 4. The special delivery relationship set. The special delivery relationship set is defined as follows:

$S T=\left\{s t_{k} \mid k=1,2, \ldots, K\right\}$

Where $S T$ is a special set of transfer relations; $s t_{k}$ is a transfer special relationship of the $k$-th state , $s t_{k}=\left(s t f_{k}, s t t_{k}\right), s t f_{k}$ is the $k$-th a failure subset of special transfer relationship, $s t t ~ k$ represents the $k$-th a testing subset of special transfer relationship.

Definition 5. Extension test model. Extension test model is defined as the following 7-tuple:

$E M=(S, F, F C, T, T C, L, S T)$ 
In this model, the fault set $F$, the test set $T$, direction edge set $L$, are expressed by the intuitive graphical; state set $S$, failure criterion set $F C$, test logic value criterion set $T C$, special transfer relationship set $S T$ are expressed by the implicit properties.

Definition 6. Extended D matrix. Extended D matrix is defined as the following threedimensional matrix:

$$
D_{I \times J \times K}=\left[d_{i j k}\right]_{I \times \backslash \times K}
$$

The three-dimensional matrix is a cube-shaped arranged by $\mathrm{I} \times \mathrm{J} \times \mathrm{K} d_{i j k}$, indicating a fault and the test's related relations under different conditions. $d_{i j k}$ is the three-dimensional matrix elements, described as the following equation:

$$
d_{i j k}= \begin{cases}1, & \text { system in } s_{k} \text { state, when } t_{j} \text { can test } f_{i} \text { fault } \\ 0, & \text { system in } s_{k} \text { state, when } t_{j} \text { cannot test } f_{i} \text { fault }\end{cases}
$$

Definition 7. Extended diagnostic tree. Extended diagnostic tree is defined as the following binary tree structure of consisting by triples:

$$
T R=(N, N R, N C)
$$

Where: $T R$ is extended diagnostic tree; $N$ is a set of the diagnostic tree node, and $N=\left\{\left.n_{p}\right|_{p}=\right.$ $1,2, \ldots, P_{\}}, n_{p}$ is the $p$ node, $P$ is the total number of nodes in the diagnosis tree; $N R$ is parent-child relationship set of the diagnosis tree node, and $N R=\left\{n r_{q} \mid q=1,2, \ldots, Q\right\} ; n r_{q}$ is the $q$ parent-child relationship of diagnosis tree node, and $n r_{q}=\left(n_{a}, n_{b}\right)$, and $n_{a}$ is parent node of diagnostic tree; $n_{b}$ is the child node of diagnostic tree; $Q$ is the total number of parent-child relationship nodes of tree; $N C$ is the branch set, and $N C=\left\{n c_{q} \mid q=1,2, \ldots, Q\right\}$, and $n c_{q}$ is the $q$ corresponding branch of parent-child relationship of the diagnosis tree node.

\section{Extended test modelling analysis algorithms}

\subsection{Model building algorithm}

Extended test modeling algorithm shown in Figure 1 below:

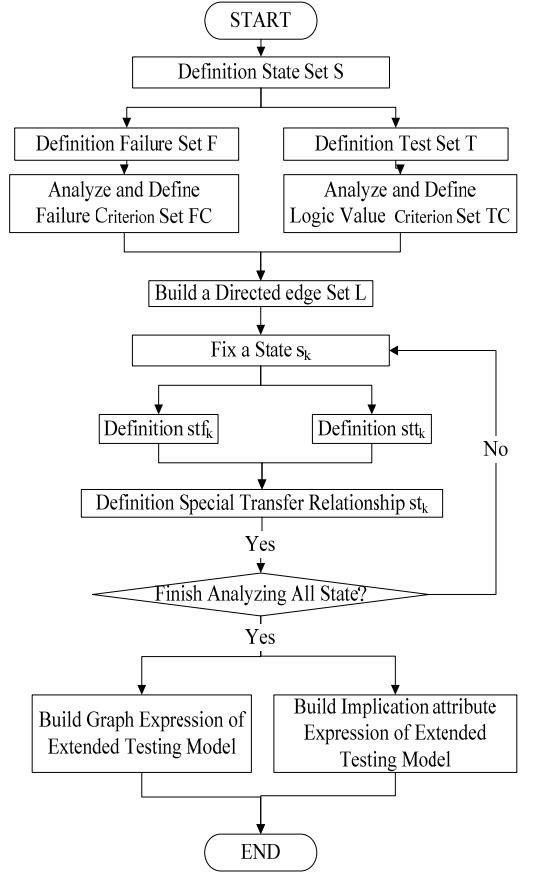

Figure1. Modeling algorithm Figure

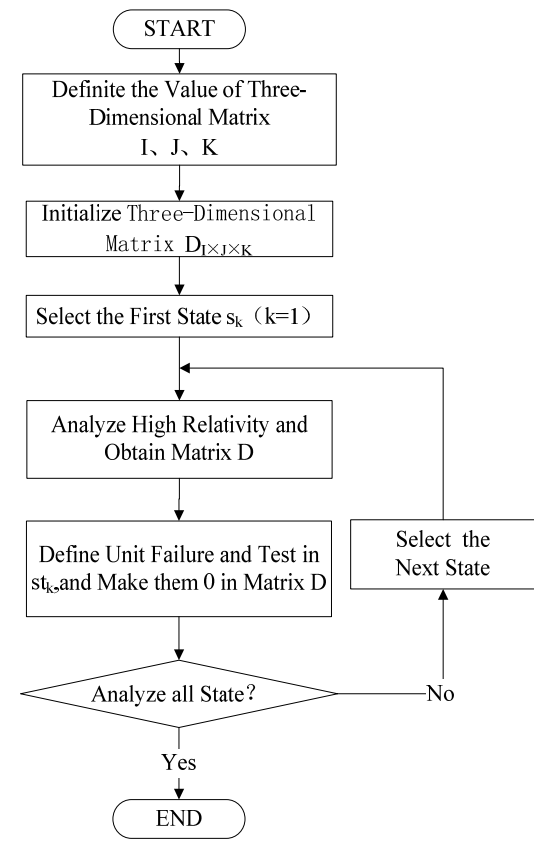

Figure2. Extended D matrix generation algorithms

1) Determine the state set $S$

According to product design, analysis its work principle, determine the product's working status, constitute the state set $S$.

2) Build extended fault $E F$ 
According to modeling requirements and reliability design data, to determine the level of modules modeling contract, the failure units constitute a set $F$. Analysis failure criterion of each unit in different state and the criterion constitute a failure set $F C$.

3) Establish extensive testing ET

According to the product testing design, determine system's testing point, build-in test and external test, which form the test set $T$. Analysis GO (pass) and NOGO (not pass) criteria of each test in each state, they constitute a set of logical value criteria, $T C$.

4) Establish directed edge set $L$

According to product design, analysis agreement between unit-level, and with all kinds of signal flow of testing relationship, determines directed edge set.

\section{Extended D matrix generation algorithm}

Extended D matrix generation method is shown in Figure 2 described:

1) To determine the dimensions of three-dimensional matrix, initialize the extended matrix $D$

According to the fault set, the test set and the state set, determine the dimension I, J, K value of the three-dimensional matrix, initialize the three-dimensional matrix.

2) Assignment of extended matrix D

(1) selected the first state $s_{k}(k=1)$ from the state set $S$, as a condition to be analyzed;

(2) According to the graphical representation of extended test model, the high-order correlation analysis is in progress, to get he D matrix in the state;

(3) According to the special transition relationship st ${ }_{k}$, determine the units' fault of the $s t f_{k}$ and the test in the $s t t k_{k}$. In the D matrix, correlation relationship of these faults and tests will set to 0;

\section{Conclusions}

Extensive test modeling method has broken the bound of the existing test methods in modeling failure criterion, simulation faults injection, failure transmission and consistency of the logical value criterion of the test. And it can describe testing information in the each state of the on-off multistate system. It can be used in many multi-state designing and analyzing work. The D matrix generated and the extended diagnosis tree provides comprehensive strategy for fault injection and diagnosis diagnostic in each state. The $\mathrm{D}$ matrix and the extended diagnosis tree generation process is very complicated, so design and development supporting software for automate is necessary.

\section{References}

[1] Ren Yueqing, Xu Lixin, based on multi-signal model of the fuse test modeling [J]. Science and Technology Review, 2010,28 (2) :67-70

[2] Guang Jin, Ye Xiaohui, Wang Hongxia. Multi-signal model in fault diagnosis [J]. Computer Measurement and Control, 2009,17 (1) :25-27

[3] Jeffrey Smith, Duane Lowenstein.Built in Test - Coverage and Diagnostics[C]// AUTOTESTCON. Anaheim,CA:IEEE,2009:169-172

[4] Somnath Deb, Charles Domagala, Sudipto Ghoshal, Ann Patterson-Hine, Richard Alena, Remote Diagnosis of the International Space Station utilizing Telemetry Data[J]. SPIE, 2008.

[5] Shijun You, Gong Jingjing, Xu Qingbo Considering the multi-fault testability modeling improved method [J]. Journal of Beijing University of Aeronautics and Astronautics, 2010,36 (3) :270-273

[6] Yang Zhiyong, Xuai Jiang, Wang Ziling. On the failure mode in multi-signal model to improve modeling study [J]. Micro-computer information, 2009,25 (4-1) :151-152 